\title{
Agroindustrial Science
}

Escuela de Ingeniería Agroindustrial

Website: http://revistas.unitru.edu.pe/index.php/agroindscience

Universidad Nacional de Trujillo

\section{Efecto de la medicina herbolaria en la calidad de vida: inventario de especies etnomedicinales y percepción del poblador de Laredo, Perú}

\author{
Effect of herbal medicine on quality of life: inventory of ethnomedical species and \\ perception of the populator of Laredo, Peru
}

\author{
José Mostacero-León* (D); Anthony J. De La Cruz-Castillo (D); Eloy Lopéz-Medina (1); \\ Armando Efraín Gil- Rivero $\mathbb{D}^{\text {; }}$ Elki T. Alfaro-Aguirre
}

Facultad de Ciencias Biológicas, Universidad Nacional de Trujillo, Perú.

\begin{abstract}
RESUMEN
Se determinó la efectividad de la medicina herbolaria y su influencia sobre la calidad de vida desde la percepción del poblador del distrito de Laredo, Trujillo, durante 2019. Se aplicó 96 entrevistas en base al Cuestionario de Salud SF-12, v2. Se inventarió 68 especies etnomedicinales, distribuidas en 67 géneros y 38 familias, siendo las Asteraceae, Lamiaceae, Fabaceae y Rosaceae las más representativas. Por su índice de valor de uso, destacan: Gentianella bicolor (IVU=0,82); Matricaria chamomilla (IVU $=0,73$ ) y Uncaria tomentosa (IVU =0,66). Los pobladores refieren sufrir 49 enfermedades, agrupadas en 11 Categorías, donde predominan las enfermedades de los Sistemas: Músculoesquelético $(\mathrm{FCl}=0,79)$, digestivo y gastrointestinal $(\mathrm{FCl}=0,79)$, nervioso $(\mathrm{FCl}=0,78)$, entre otras. Se concluye que la medicina herbolaria tiene una efectividad del $100 \%$; puesto que curan un $68,02 \%$ y alivian un $32,08 \%$ de la población y el $77,41 \%$ atribuye haber mejorado su calidad de vida.
\end{abstract}

Palabras clave: efectividad; medicina herbolaria; calidad de vida; especies etnomedicinales; medicina tradicional.

\section{ABSTRACT}

The effectiveness of the herbal medicine and its influence on the quality of life from the perception of the population of the district of Laredo, Trujillo, was determined during 2019. 96 interviews were applied based on the Health Questionnaire SF-12, v2. 68 ethnomedical species were inventoried, distributed in 67 genera and 38 families, being the Asteraceae, Lamiaceae, Fabaceae and Rosaceae the most representative. For its use value index include: Gentianella bicolor (IVU $=0.82$ ); Matricaria chamomilla (IVU $=0.73$ ) and Uncaria tomentosa (IVU $=0.66$ ). The residents refer 49 diseases, grouped into 11 categories, where predominate diseases of the Systems: Musculoskeletal $(\mathrm{FCl}=0.79)$, digestive and gastrointestinal $(\mathrm{FCl}=0.79)$, nervous $(\mathrm{FCl}=0.78)$, among others. It is concluded that the herbal medicine has $100 \%$ effectiveness; since they cure $68.02 \%$ and relieve $32.08 \%$ of the population and $77.41 \%$ attribute having improved their quality of life.

Keywords: effectiveness; herbal medicine; quality of life; ethnomedical species; traditional medicine.

\section{Introducción}

No cabe duda que la medicina tradicional en general, y la medicina herbolaria en especial, ha jugado, juega y jugará un rol preponderante en el tratamiento de las más diversas enfermedades y/o dolencias que ha sufrido el hombre a través de su historia; a tal punto que se estima que más del $80 \%$ de la población mundial, recurre a estos importantes recursos, para mejorar su calidad de vida (Annan y Houghton, 2007; Mostacero et al., 2011; Garzón, 2016).
En Perú esta práctica fue muy bien dirigida, desde tiempos inmemoriales por los "Hampicamayoc", "Oquetlupuc" y "Sircac"; antiguos médicos y boticarios, capaces de realizar un eficaz y correcto uso de las plantas medicinales; preparadas como: infusión, jugos, decocciones, extractos, tisanas, maceraciones, tinturas, polvos, pastas, emplastos, cataplasmas, cremas, ungüentos, aceites esenciales entre otros; y administradas en forma de: baños, gárgaras, bebidas, lavados vaginales, inhalaciones, tópica, entre otros; constituyéndose 
este conocimiento en un legado del Perú para el mundo (Mostacero et al., 2011; Pascual et al., 2014; Salaverry y Cabrera, 2014).

A pesar de la enorme importancia de estos recursos, la sabiduría ancestral de ellos, se ha ido perdiendo paulatinamente en el tiempo, a tal punto que corre el riesgo de desaparecer sino aten-demos, recuperamos y estructuramos el conocimiento aún presente en los "curanderos", "abuelitas", "sabedores de plantas" y el resto de integrantes de las Comunidades Andinoamazónicas; rurales, sub urbanas y urbanas; trabajo de recuperación de conocimientos que atañe sobre todo a los Antropólogos, Etnobotánicos y Médicos y así tener el insumo necesario para la realización de trabajos de tipo fitoquímico, farmacológico y de ensayos clínicos, que no sólo nos permitan conocer los principios activos 0 metabolitos secundarios; sino también la acción farmacológica que tienen éstos, en el tratamiento de las diferentes enfermedades (Bussmann y Glenn, 2010; Mostacero et al., 2011).

Referente a los aspectos de taxonomía y etnobotánica, afortunadamente se conocen varios trabajos en el Perú, que reportan no sólo la gran cantidad de especies que tenemos, formas de preparación y administración, así como algunos aspectos fitogeográficos; que podemos analizarlo en la bibliografía que al respecto se ha consultado y que está a entera disposición (Sagástegui, 1995; Bussmann y Sharon, 2006; Mostacero et al., 2011; Rengifo y Vargas, 2013; Castillo et al., 2017; Hurtado y Albán, 2018; Mostacero et al., 2019).

En este sentido, está demostrado que las plantas medicinales en el Perú son utilizadas por cerca de las 2/3 parte de la población. Sin embargo, su uso puede ser más amplio, correcto y eficaz, si la investigación los validara científicamente, para contar con recursos no sólo de bajo costo, sino también accesibles y eficaces para los pobladores, más excluidos y pobres, quienes tienen escaso acceso a la medicina oficial y académica (Bussmann y Sharon, 2006; Mostacero et al., 2011).

Por otro lado, la etnobotánica nos habla de muchas formas sobre la eficacia que tienen las plantas medicinales para la cura y/o alivio de diferentes dolencias $y / 0$ enfermedades y que consecutivamente contribuyen a mejorar la calidad de vida de las personas, a pesar de que este término abarque aspectos no relacionados tan estrechamente con la salud, como los aspectos culturales, políticos y sociales; vaguedad conceptual que estimuló a tomar el término "Calidad de vida relacionada a la salud" (CVRS) ; para referirse específicamente a la salud de la persona, nombrando únicamente aspectos de la vida relacionadas a la salud (Bradburn, 1969; Glatzer y Zapf, 1984; Fernández et al., 2010).

Actualmente se han establecido metodologías para evaluar la efectividad de la medicina herbolaria y el papel que juega en la CVRS de las personas; es así que se conocen los trabajos de Ware y Sherbourne (1992); Ware et al. (1993); Ware et al. (1996); Consiglio y Belloso (2003); Vilagut et al. (2005); Schwartzmann (2003); y practicada en el Perú por Bocanegra et al. (2011) en la Comunidad de Curgos, Sánchez Carrión, Perú.

Por lo descrito en los párrafos anteriores y al no encontrarse reporte alguno sobre la evaluación de la efectividad de la medicina Herbolaria en la Provincia de Trujillo y más específicamente en el distrito de Laredo, la presente investigación se orientó a determinar la efectividad de la Medicina Herbolaria sobre la calidad de vida desde la percepción del poblador del distrito de Laredo, Trujillo, durante 2019, como una forma de revalorar, incentivar y promover el uso de las plantas medicinales, así como, fomentar la importancia que tienen en la mejora de la calidad de vida.

\section{Material y métodos}

Lugar de ejecución y muestra

La fuente de datos para esta investigación procedió de las entrevistas realizadas en los mercados del distrito de Laredo, provincia de Trujillo, La Libertad, Perú (Figura 1), ubicado entre $8^{\circ} 00^{\prime} 30^{\prime \prime}$ y $8^{\circ} 08^{\prime} 30^{\prime \prime}$ L. S. y entre $80^{\circ} 50^{\prime} 00^{\prime \prime}$ y 79015'00" L. O. (CIPADEL, 1992).

Se aplicaron 96 entrevistas, muestra estadística que se calculó mediante la fórmula que considera población finita y varianza desconocida:

$$
\mathrm{n}=\frac{\mathrm{N} Z^{2} p q}{d^{2}(N-1)+Z^{2} p q}
$$

\section{Bocanegra (2011);}

Dónde:

$\mathrm{n}$ : Tamaño de la muestra;

$\mathrm{N}$ : Población del estudio;

p: Proporción de éxito de la característica de interés, igual a $50 \%(0,5)$;

q: 1 - $\mathrm{P}=$ complemento de $P$;

Z: Coeficiente de confiabilidad al $95 \%$ igual a 1,96;

d: Tolerancia de error permisible en la investigación $=0,1(10 \%)$. 


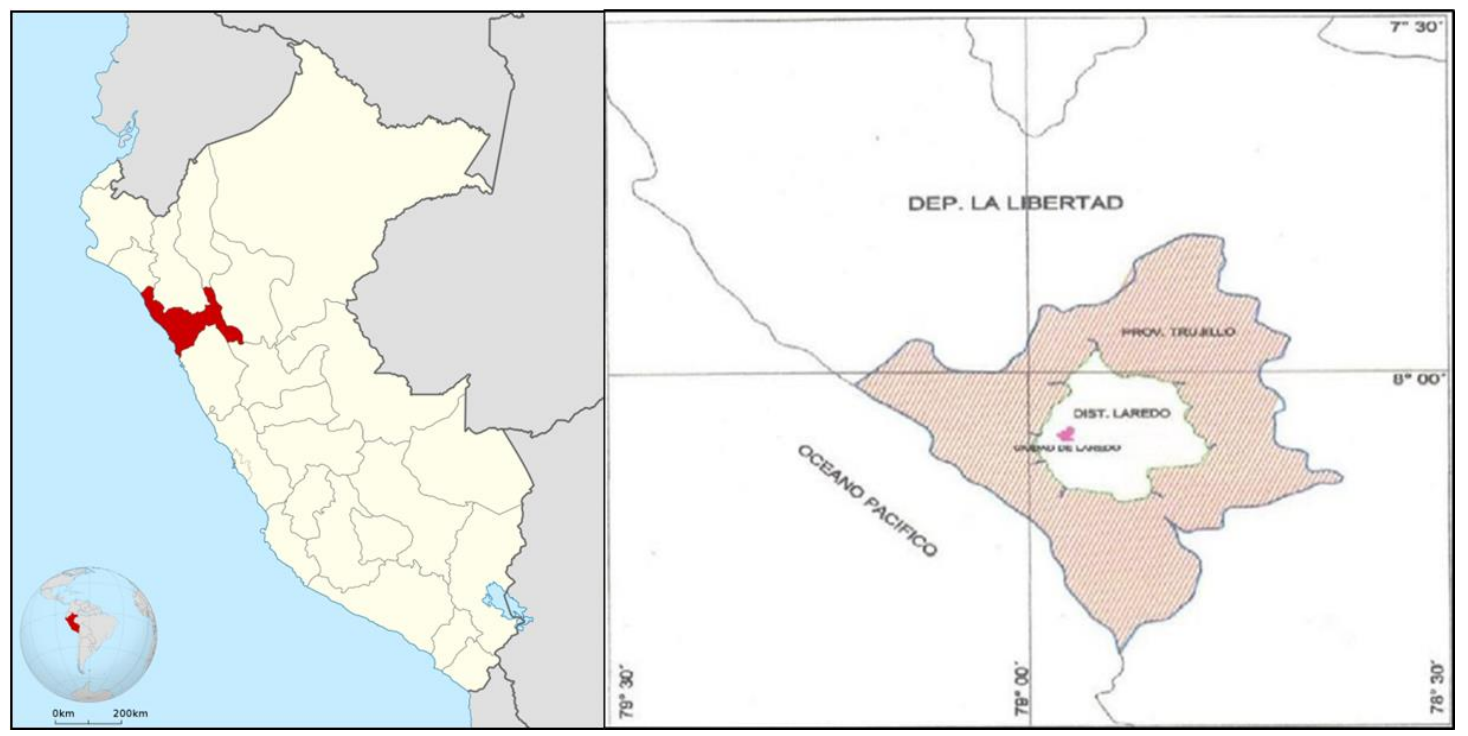

Figura 1. Ubicación del distrito de Laredo en la provincia de Trujillo. Fuente: Peláez y Yuto (2001).

\section{Técnica e instrumento de recolección de datos}

Se utilizó la técnica de la entrevista semiestructurada, la misma que consta de 3 partes: la primera, que abarca los datos generales del(a) entrevistado(a), con preguntas sobre factores sociodemográficos. La segunda, aporta información sobre efectividad de la medicina herbolaria, con preguntas sobre la percepción de los individuos respecto al nivel de efectividad del tratamiento recibido y la tercera parte, que brinda información sobre la calidad de vida relacionada con la salud, es decir buscando conocer la percepción de cada individuo, respecto a su estado posterior al último tratamiento concluido, basado en el Cuestionario de Salud SF-12,v2 modificado por Bocanegra et al. (2009).

\section{Análisis de datos}

La información recabada, fue organizada en Tablas previamente complementada con la información presentada en tesis, artículos científicos y libros sobre la materia (Mostacero et al., 2011). La calidad de vida se determinó teniendo en cuenta 8 dimensiones o escalas: Salud General, Función Física, Rol Físico, Dolor Corporal, Vitalidad, Función Social, Rol Emocional y Salud Mental (Ware et al., 1993; Ware et al., 1996), las mismas que fueron evaluadas por separado; considerando buena CVRS a la que supere el $50 \%$ de la percepción positiva del entrevistado, y mala CVRS, a aquella que no llegue al $50 \%$.

También se determinó el índice de valor de uso de cada especie (IVU), el mismo que expresa la importancia 0 valor cultural de cada especie determinada para todos los entrevistados, según fórmula propuesta por Zambrano et al. (2015):

$$
V U_{s}=\frac{V U_{i s}}{N_{s}}
$$

Donde: $V U_{i s}$ es el valor de uso de la especie por cada informante, y $N_{S}$ es el número de informantes para cada especie.

Por último, se determinó el índice de Factor de Consenso del Informante $(\mathrm{FCl})$, que mide la existencia 0 no de un consenso entre los entrevistados, en cuanto al uso de especies medicinales sobre categorías particulares de enfermedades; siguiendo la fórmula propuesta por Heinrich et al. (2009):

$$
F C I=\frac{n_{r u}-n_{t}}{n_{r u}-1}
$$

Donde: $n_{r u}$ es el número de informes de uso para una categoría de dolencia particular, y $n_{t}$ es el número de especies medicinales utilizadas para la categoría de dolencia.

\section{Identificación de la flora etnomedicinal}

Las plantas medicinales mencionadas durante la realización de las entrevistas semi- estructuradas aplicadas; fueron colectadas, herborizadas y transportadas al Herbarium Truxillense de la Universidad Nacional de Trujillo (H.U.T.), donde se realizó la determinación taxonómica, tanto por comparación con el material registrado en este Herbario, como por el asesoramiento de los investigadores expertos de esta institución; también se aplicó el corrido de claves taxonómicas y la bibliografía especializada de Brako y Zarucchi (1993) y Mostacero et al. (2009), y a su vez confirmada en los portales de Trópicos y The Plant List. 


\section{Aspectos éticos}

La presente investigación, contó con el consentimiento de las personas que participaron en las entrevistas; respetando el derecho a la privacidad de cada voluntario entrevistado.

\section{Resultados y discusión}

Estos resultados también guardan cierta similitud con los trabajos que resaltan la importancia de la flora peruana; como las investigaciones de Picard y Villar (1982), Pretell et al. (1985), Sagástegui (1995); y que están relacionados sobre todo a la taxonomía; dejando de lado los caracteres etnomedicinales de las mismas; al contrario de esta investigación que estuvo dirigido a determinar la eficacia y problemática real en la calidad de vida que brindan las plantas medicinales en la sociedad.

En la Tabla 1, se presentan los resultados referentes a: nombre científico, familia y nombre común; y los aspectos etnomedicinales: parte utilizada de la planta, tipo de preparado, modo de aplicación, usos e índice de valor de uso, de 68 especies utilizadas para la cura y/o alivio de las enfermedades y/o dolencias que aqueja el poblador del distrito de Laredo; los que se distribuyen en 67 géneros y 38 familias; siendo, las Asteraceae (13 especies), Lamiaceae (6 especies), Fabaceae (4 especies) y Rosaceae (3 especies) las más representativas por su número de especies (Figura 2); concordando con lo descrito por Bussmann y Sharon (2006),Vera y Sánchez (2016), Castañeda y Albán (2016), De la Cruz y Mostacero (2019), Lara et al. (2019), Ortega-Cala et al. (2019), quienes ratifican el hecho de que tanto las familias Asteraceas y Lamiaceas lideran la representatividad en la flora etnomedicinal, debido a su amplia distribución, cantidad de especies, así como a su riqueza en muchos metabolitos secundarios, como los aceites esenciales que les confiere atributos para combatir problemas gastrointestinales, respiratorios, del sistema nervioso, así como por su poder antiinflamatorio, entre otros; es así Es así que estos primeros resultados se suman a los reportados por Mostacero et al. (2009), Mostacero et al. (2011) y De la Cruz y Mostacero (2019).

En la misma Tabla, se reporta el índice de valor de uso, o estatus preferencial que el poblador le atribuye a cada especie en el tratamiento de sus enfermedades, donde hay que considerar que valores mayores a 0,5 indican un mayor grado de importancia de la especie. Así: Gentianella bicolor "corpus huay" (IVU=0,82); Matricaria chamomilla "manzanilla" (IVU= 0,73); Uncaria tomentosa "uña de gato" (IVU= 0,66); Linum usitatissimum "linaza" (IVU= 0,63); Solanum tuberosum "papa" (IVU= 0,59); Equisetum bogotense "cola de caballo" (IVU= 0,56); Opuntia ficus-indica "tuna" (IVU= $0,55)$ y Aloe vera "sábila" (IVU=0,53); indican que son las especies de mayor valor de uso que el resto de las 68 especies; resultados similares con lo descrito por autores como Lara et al. (2019), Bocanegra et al. (2011); pero mostrando diferencias con lo reportados por Zambrano et al. (2015), quienes cataloga para Ecuador a Cymbopogon citratus "hierba luisa", Origanum vulgare "orégano", Mentha sativa "hierba buena", Mentha rotundifolia "menta", Melissa officinalis "toronjil y Dysphania ambrosioides "paico"; como las especies más importantes; lo que probablemente se deba a factores biológicos, ecológicos y sociales que influyen en la normal distribución de las mismas.

Por otro lado, Bussmann y Sharon (2015) catalogan para el Norte del Perú, 510 plantas de uso medicinal. Laredo es un distrito que representa sólo el $13 \%$ de la población del Norte peruano. Entonces las 68 especies reflejan un número representativo y acorde con los hallazgos de esta investigación; teniendo en cuenta que el tipo de estudio es similar no sólo por el área geográfica, sino también por la metodología utilizada basada en entrevistas a las personas beneficiadas de las plantas medicinales, que recurren a los mercados donde los "herbolarios", "emolienteros" y otras personas dedicadas a su comercialización, que proporcionan información sobre gran cantidad de plantas medicinales que se comercializan y se consumen, provenientes de la colección abundante y que sin duda está atentando con su conservación; pues muchas de estas especies están prácticamente en peligro de extinción, sin que hasta la fecha se implemente un verdadero plan de conservación para evitar su extinción con perjuicio de negar a las futuras generaciones, estos importantes recursos, tal como advierten Mostacero et al. (2007); Mostacero et al. (2011); quienes dejan establecida que esta problemática se resolvería atendiendo al conocimiento ecológico, fitogeográfico, fenológico y de propagación permanente de estos recursos.

En la Tabla 2 se detalla 49 enfermedades y/o dolencias manifestada por los pobladores del distrito de Laredo, las mismas que han sido agrupadas en 11 categorías de acuerdo a The International Classification of Diseases used by the World 
Health Organization (ICD, 2017), y que según esta categorización en Laredo, están representadas las enfermedades de los Sistemas: Músculoesquelético $(\mathrm{FCl}=0,79)$, digestivo y gastrointestinal $(\mathrm{FCl}=0,79)$, del nervioso $(\mathrm{FCl}=0,78)$, del cardiovascular $(\mathrm{FCl}=0,75)$, del urinario $(\mathrm{FCl}=0,75)$, para Síntomas o padecimientos generales $(\mathrm{FCl}=0,75)$, las del respiratorio $(\mathrm{FCl}=0,74)$, las del reproductivo $(\mathrm{FCl}=0,73)$ y del grupo de las crónico-degenerativas $\quad(\mathrm{FCl}=0,70) . \quad$ Estos resultados sólo encuentran comparación con los de Huamantupa et al. (2011), Bocanegra et al. (2011), Bussmann y Sharon (2015), Mostacero et al. (2019); quienes detallan por lo menos en porcentajes que las enfermedades digestivas, urinarias y respiratorias son las más frecuentes en diferentes partes del Perú; de lo que se infiere que este trabajo es más amplio y completo.

Tabla 1

Caracteres taxonómicos, etnobotánicos e Índice de valor de uso de la flora utilizada por el poblador del distrito de Laredo, Trujillo, La Libertad

\begin{tabular}{|c|c|c|c|c|c|c|c|c|}
\hline $\mathrm{N}$ & NOMBRE CIENTÍFICO & $\begin{array}{l}\text { NOMBRE } \\
\text { COMÚN }\end{array}$ & $\begin{array}{l}\text { PARTE } \\
\text { UTILIZADA }\end{array}$ & $\begin{array}{c}\text { TIPO DE } \\
\text { PREPARADO }\end{array}$ & $\begin{array}{l}\text { MODO } \\
\text { DE } \\
\text { APLIC } \\
\text { ACIÓN }\end{array}$ & USO & RU & IVU \\
\hline 1 & $\begin{array}{l}\text { Achyrocline alata (Kunth) DC. } \\
\text { ASTERACEAE }\end{array}$ & "paigosa" & tallo y hojas & Cocimiento & Oral & $\begin{array}{l}\text { contra el colesterol, depuradora de } \\
\text { sangre. }\end{array}$ & 9 & 0,09 \\
\hline 2 & $\begin{array}{l}\text { Adiantum subvolubile Mett. } \\
\text { ex Kuhn } \\
\text { PTERIDACEAE }\end{array}$ & "culantrillo" & rizomas & Cocimiento & Oral & $\begin{array}{l}\text { acción colagoga,depurativa y } \\
\text { sedante }\end{array}$ & 1 & 0,01 \\
\hline 3 & $\begin{array}{l}\text { Aloe vera (L.) Burm.f. } \\
\text { XANTHORRHOEACEAE }\end{array}$ & "sábila" & hojas & $\begin{array}{l}\text { extracto, } \\
\text { emplasto }\end{array}$ & $\begin{array}{l}\text { Oral y } \\
\text { tópica }\end{array}$ & $\begin{array}{l}\text { acción antiasmática, desinflamante y } \\
\text { vulnerario }\end{array}$ & 51 & 0,53 \\
\hline 4 & $\begin{array}{l}\text { Aloysia citriodora Palau } \\
\text { VERBENACEAE }\end{array}$ & "cedrón" & tallo y hojas & $\begin{array}{l}\text { Infusión y } \\
\text { cocimiento }\end{array}$ & Oral & $\begin{array}{l}\text { acción estomacal y propiedades } \\
\text { digestivas. }\end{array}$ & 12 & 0,13 \\
\hline 5 & $\begin{array}{l}\text { Ambrosia peruviana Willd. } \\
\text { ASTERACEAE }\end{array}$ & $\begin{array}{l}\text { "marco", } \\
\text { "altamisa" }\end{array}$ & tallo y hojas & Cocimiento & Oral & $\begin{array}{l}\text { acción antirreumática, } \\
\text { antiespasmódica, contra la } \\
\text { dismenorrea. }\end{array}$ & 24 & 0,25 \\
\hline 6 & $\begin{array}{l}\text { Annona muricata L. } \\
\text { ANNONACEAE }\end{array}$ & $\begin{array}{l}\text { "guanában } \\
\text { a" }\end{array}$ & hojas & $\begin{array}{l}\text { Infusión, } \\
\text { Cocimiento }\end{array}$ & Oral & $\begin{array}{l}\text { acción desinflamante del aparato } \\
\text { reproductor. }\end{array}$ & 9 & 0,09 \\
\hline 7 & $\begin{array}{l}\text { Argyrochosma nivea (Poir.) } \\
\text { Windham } \\
\text { PTERIDACEAE }\end{array}$ & "cuti cuti" & $\begin{array}{l}\text { Frondas } \\
\text { (hojas) }\end{array}$ & $\begin{array}{l}\text { Infusión y } \\
\text { cocimiento }\end{array}$ & Oral & acción antidiabética. & 22 & 0,23 \\
\hline 8 & $\begin{array}{l}\text { Artemisia absinthium L. } \\
\text { ASTERACEAE }\end{array}$ & "ajenjo" & $\begin{array}{l}\text { toda la } \\
\text { planta }\end{array}$ & Infusión. & Oral & $\begin{array}{l}\text { acción antidiarreica, digestiva y } \\
\text { vulneraria. }\end{array}$ & 2 & 0,02 \\
\hline 9 & $\begin{array}{l}\text { Baccharis sagittalis (Less.) } \\
\text { DC. ASTERACEAE }\end{array}$ & "carqueja" & tallo y hojas & Cocimiento. & Oral & $\begin{array}{l}\text { acción depuritiva, contra dolores } \\
\text { reumáticos. }\end{array}$ & 16 & 0,17 \\
\hline $\begin{array}{l}1 \\
0\end{array}$ & $\begin{array}{l}\text { Bidens pilosa L. } \\
\text { ASTERACEAE }\end{array}$ & $\begin{array}{l}\text { "amor } \\
\text { seco" }\end{array}$ & $\begin{array}{l}\text { toda la } \\
\text { planta }\end{array}$ & $\begin{array}{l}\text { Infusión, } \\
\text { Cocimiento }\end{array}$ & Oral & acción colagoga y diuretica. & 40 & 0,42 \\
\hline $\begin{array}{l}1 \\
1\end{array}$ & Bixa orellana L. BIXACEAE & "achiote" & hoja & $\begin{array}{l}\text { Infusión, } \\
\text { Cocimiento }\end{array}$ & Oral & $\begin{array}{l}\text { acción antiinflamatoria, contra la } \\
\text { prostatitis. }\end{array}$ & 33 & 0,34 \\
\hline $\begin{array}{l}1 \\
2\end{array}$ & $\begin{array}{l}\text { Buddleja coriacea Remy } \\
\text { SCROPHULARIACEAE }\end{array}$ & $\begin{array}{c}\text { "flor } \\
\text { blanca" }\end{array}$ & tallo y hojas & $\begin{array}{l}\text { Infusión y } \\
\text { cocimiento }\end{array}$ & Oral & acción colagoga y sedante & 1 & 0,01 \\
\hline $\begin{array}{l}1 \\
3\end{array}$ & $\begin{array}{l}\text { Caesalpinia spinosa (Molina) } \\
\text { Kuntze } \\
\text { FABACEAE }\end{array}$ & $\begin{array}{l}\text { "tara", } \\
\text { "taya" }\end{array}$ & $\begin{array}{l}\text { fruto y } \\
\text { semilla }\end{array}$ & Otros (gárgaras) & $\begin{array}{l}\text { Gargar } \\
\text { ismo }\end{array}$ & acción desinflamante y vulneraria & 24 & 0,25 \\
\hline $\begin{array}{l}1 \\
4\end{array}$ & $\begin{array}{l}\text { Campyloneurum } \\
\text { angustifolium (Sw.) Fée } \\
\text { POLYPODIACEAE }\end{array}$ & "calaguala" & rizomas & Cocimiento & Oral & $\begin{array}{l}\text { acción colagoga,desinflamante y } \\
\text { espasmolitica }\end{array}$ & 3 & 0,03 \\
\hline $\begin{array}{l}1 \\
5\end{array}$ & $\begin{array}{l}\text { Cichorium intybus L. } \\
\text { ASTERACEAE }\end{array}$ & $\begin{array}{l}\text { "achicoria", } \\
\text { "amargón" }\end{array}$ & hoja & Cocimiento. & Oral & $\begin{array}{l}\text { contra afecciones biliares, de la } \\
\text { vesícula, antidiarreica, depurativa } \\
\text { de la sangre, colagoga y colerética. }\end{array}$ & 43 & 0,45 \\
\hline $\begin{array}{l}1 \\
6\end{array}$ & $\begin{array}{l}\text { Citrus aurantiifolia (Christm.) } \\
\text { Swingle } \\
\text { RUTACEAE }\end{array}$ & "lima" & fruto & jugo & Oral & $\begin{array}{l}\text { acción digestiva, calmante y } \\
\text { antiespasmódica. }\end{array}$ & 34 & 0,35 \\
\hline $\begin{array}{l}1 \\
7\end{array}$ & $\begin{array}{l}\text { Citrus limon (L.) Osbeck } \\
\text { RUTACEAE }\end{array}$ & "limón" & fruto & jugo & Oral & $\begin{array}{l}\text { combate la conjuntivitis, gripe, } \\
\text { asma, dolor de cabeza. }\end{array}$ & 31 & 0,32 \\
\hline $\begin{array}{l}1 \\
8\end{array}$ & $\begin{array}{l}\text { Clinopodium pulchellum } \\
\text { (Kunth) Govaerts } \\
\text { LAMIACEAE }\end{array}$ & "panisara" & tallo y hojas & Infusión & Oral & acción carminativa y desinflamante & 30 & 0,31 \\
\hline $\begin{array}{l}1 \\
9\end{array}$ & $\begin{array}{l}\text { Crescentia cujete L. } \\
\text { BIGNONIACEAE }\end{array}$ & "tutumo" & fruto & Maceración & Oral & $\begin{array}{l}\text { acción febrífuga, purgativa y } \\
\text { espectorante, contra las } \\
\text { enfermedades respiratorias. }\end{array}$ & 8 & 0,08 \\
\hline $\begin{array}{l}2 \\
0\end{array}$ & $\begin{array}{l}\text { Cymbopogon citratus (DC.) } \\
\text { Stapf POACEAE }\end{array}$ & $\begin{array}{l}\text { "hierba } \\
\text { luisa" }\end{array}$ & hojas & $\begin{array}{l}\text { Infusión, } \\
\text { cocimiento }\end{array}$ & Oral & acción carminativa y espasmolíticas & 11 & 0,11 \\
\hline $\begin{array}{l}2 \\
1\end{array}$ & $\begin{array}{l}\text { Desmodium molliculum } \\
\text { (Kunth) DC. } \\
\text { FABACEAE }\end{array}$ & $\begin{array}{l}\text { "pie de } \\
\text { perro" }\end{array}$ & $\begin{array}{l}\text { toda la } \\
\text { planta }\end{array}$ & Infusión & Oral & acción diurética y desinflamante & 45 & 0,47 \\
\hline $\begin{array}{l}2 \\
2\end{array}$ & $\begin{array}{l}\text { Dioscorea moyobambensis } \\
\text { R.Knuth }\end{array}$ & $\begin{array}{c}\text { "papa } \\
\text { semitona" }\end{array}$ & raiz & Cocimiento & Oral & $\begin{array}{l}\text { acción colagoga,carminativa y } \\
\text { digestiva }\end{array}$ & 15 & 0,16 \\
\hline
\end{tabular}




\section{DIOSCOREACEAE}

2 Equisetum bogotense Kunth

3 EQUT

2 Erythroxylum coca Lam.

4 ERYTHROXYLACEAE

2 Eucalyptus globulus Labill.

5 MYRTACEAE

2 Foeniculum vulgare Mill.

6 APIACEAE

Gentianella bicolor (Wedd.)

Fabris ex J.S.Pringle

GENTIANACEAE

2 Geranium ruizii Hieron.

GERANIACEAE

2 Linum usitatissimum L.

LINACEAE

3 Marrubium vulgare L.

LAMIACEAE

3 Matricaria chamomilla L.

ASTERACEAE

3 Mauria heterophylla Kunth

2 ANACARDIACEAE

3 Melissa officinalis $\mathrm{L}$.

3 LAMIACEAE

Mentha $\times$ piperita $L$

4 LAMIACEAE

Minthostachys mollis (Benth.)

Griseb.

LAMIACEAE

3 Morinda citrifolia L.

6 RUBIACEAE

Musa $\times$ paradisiaca $\mathrm{L}$

7 MUSACEAE

Myrcianthes oreophila (Diels)

McVaugh

MYRTACEAE

Nasturtium officinale $\mathrm{R} . \mathrm{Br}$.

BRASSICACEAE

4 Oenothera rosea L'Hér. ex

0 Aiton ONAGRACEAE

4 Opuntia ficus-indica (L.) Mill.

1 CACTACEAE

4 Passiflora edulis Sims

2 PASSIFLORACEAE

Peperomia inaequalifolia Ruiz

\& Pav

PIPERACEAE

Phyllanthus niruri L.

PHYLLANTHACEAE

Physalis peruviana $\mathrm{L}$.

5 SOLANACEAE

4 Picrosia longifolia D.Don

6 ASTERACEAE

Piper aduncum $L$

PIPERACEAE

4 Plantago major $\mathrm{L}$.

8 PLANTAGINACEAE

Polylepis incana Kunth

ROSACEAE

5 Psoralea glandulosa $\mathrm{L}$.

FABACEAE

5 Rosmarinus officinalis $\mathrm{L}$.

LAMIACEAE

Rubus roseus Poir.

2 ROSACEAE

5 Sambucus peruviana Kunth

ADOXACEAE

\begin{tabular}{|c|c|c|c|c|c|c|}
\hline $\begin{array}{l}\text { "cola de } \\
\text { caballo" }\end{array}$ & $\begin{array}{l}\text { toda la } \\
\text { planta }\end{array}$ & $\begin{array}{l}\text { Infusión, } \\
\text { Cocimiento }\end{array}$ & Oral & $\begin{array}{l}\text { acción antidiarreica, digestiva, } \\
\text { depurativa, desinflamante y } \\
\text { colagoga. }\end{array}$ & 54 & 0,56 \\
\hline "coca" & hojas & Infusión & Oral & $\begin{array}{l}\text { acción antidiarreica, antiasmática, } \\
\text { carminativa y digestiva. }\end{array}$ & 26 & 0,27 \\
\hline "eucalipto" & hojas & $\begin{array}{l}\text { Infusión y } \\
\text { cocimiento }\end{array}$ & $\begin{array}{l}\text { Oral y } \\
\text { baños }\end{array}$ & acción antiasmatica & 38 & 0,40 \\
\hline "hinojo" & $\begin{array}{l}\text { frutos y } \\
\text { semillas }\end{array}$ & Infusión. & Oral & acción calmante & 14 & 0,15 \\
\hline $\begin{array}{l}\text { "corpus } \\
\text { huay" }\end{array}$ & $\begin{array}{l}\text { toda la } \\
\text { planta }\end{array}$ & $\begin{array}{l}\text { Infusión, } \\
\text { Cocimiento }\end{array}$ & Oral & acción colagoga y vulnerativa & 79 & 0,82 \\
\hline $\begin{array}{l}\text { "pasuchac } \\
\text { a" }\end{array}$ & raíz & Cocimiento & Oral & acción antidiabética. & 24 & 0,25 \\
\hline "linaza" & semilla & Cocimiento & Oral & acción antidiarreica y desinflamante & 60 & 0,63 \\
\hline $\begin{array}{l}\text { "cordón de } \\
\text { muerto" }\end{array}$ & tallo y hojas & Cocimiento & Oral & $\begin{array}{l}\text { estimulante de la secreción biliar, } \\
\text { estomacal, antiséptica, antidiarreica. }\end{array}$ & 2 & 0,02 \\
\hline $\begin{array}{l}\text { "manzanill } \\
\text { a" }\end{array}$ & hoja y flores & Infusión. & Oral & $\begin{array}{l}\text { acción carminativa, espasmolítica, } \\
\text { antidiarreica digestiva, sedante y } \\
\text { vulneraria. }\end{array}$ & 70 & 0,73 \\
\hline "trinidad" & hojas & $\begin{array}{l}\text { Cocimiento, } \\
\text { pastas }\end{array}$ & Oral & $\begin{array}{l}\text { acción desinfectante, combate las } \\
\text { caries y vulneraria. }\end{array}$ & 15 & 0,16 \\
\hline "toronjil" & tallo y hojas & $\begin{array}{l}\text { Infusión, } \\
\text { Cocimiento }\end{array}$ & Oral & acción carminativa y espasmolíticas & 24 & 0,25 \\
\hline "menta" & $\begin{array}{l}\text { toda la } \\
\text { planta }\end{array}$ & Infusión & Oral & $\begin{array}{l}\text { acción antidiarreica, emenagoga, } \\
\text { estomáquica y sedante. }\end{array}$ & 43 & 0,45 \\
\hline "muña" & tallo y hojas & $\begin{array}{l}\text { Infusión, } \\
\text { Cocimiento }\end{array}$ & Oral & acción estimulante y carminativa. & 43 & 0,45 \\
\hline "noni" & fruto & extracto & Oral & acción desinflamnte. & 2 & 0,02 \\
\hline "plátano" & escapo & extracto & Oral & acción vulneraria & 12 & 0,13 \\
\hline "unquia" & hojas & Cocimiento & Oral & $\begin{array}{l}\text { acción antiinflamatoria, estomáquica } \\
\text { y vulneraria. }\end{array}$ & 24 & 0,25 \\
\hline "berro" & $\begin{array}{l}\text { hoja y } \\
\text { hierba }\end{array}$ & Ensalada & Oral & acción colagoga y refrigerante & 30 & 0,31 \\
\hline $\begin{array}{l}\text { "chupasan } \\
\text { gre", } \\
\text { "iglesia" }\end{array}$ & tallo y hojas & Emplasto & Tópica & $\begin{array}{l}\text { contra heridas y raspaduras, } \\
\text { inflamaciones cutáneas, dermatosis. }\end{array}$ & 3 & 0,03 \\
\hline "tuna" & cladodio & extracto & Oral & $\begin{array}{l}\text { contra desórdenes digestivos, } \\
\text { afecciones de la piel y } \\
\text { reconstituyente. }\end{array}$ & 53 & 0,55 \\
\hline "maracuya & fruto & Jugo & Oral & $\begin{array}{l}\text { contra la hipertensión y } \\
\text { cardiotónico, acción sedante. }\end{array}$ & 27 & 0,28 \\
\hline "congona" & tallo y hojas & Maceración & Oral & acción sedante & 14 & 0,15 \\
\hline $\begin{array}{l}\text { "chancapie } \\
\text { dra" }\end{array}$ & tallo y hojas & Cocimiento. & Oral & $\begin{array}{l}\text { acción diurética y para disolver } \\
\text { cálculos renales y del hígado. }\end{array}$ & 11 & 0,11 \\
\hline $\begin{array}{l}\text { "aguayman } \\
\text { to" }\end{array}$ & frutos & jugos, extractos & Oral & acción antigripal y digestiva. & 3 & 0,03 \\
\hline $\begin{array}{l}\text { "achicoria", } \\
\text { "chicoria" }\end{array}$ & hoja & Cocimiento. & Oral & $\begin{array}{l}\text { contra afecciones biliares, de la } \\
\text { vesícula, antidiarreica, depurativa } \\
\text { de la sangre, colagoga y colerética. }\end{array}$ & 44 & 0,46 \\
\hline "matico" & hojas & $\begin{array}{l}\text { Infusión y } \\
\text { cocimiento }\end{array}$ & Oral & $\begin{array}{l}\text { acción desinflamante, antigripal y } \\
\text { sedante }\end{array}$ & 48 & 0,50 \\
\hline "llantén" & hoja & $\begin{array}{l}\text { Infusión, } \\
\text { cocimiento y } \\
\text { emplasto }\end{array}$ & $\begin{array}{l}\text { Oral y } \\
\text { tópica }\end{array}$ & acción desinflamante y sedante & 15 & 0,16 \\
\hline "quinual" & hojas & Cocimiento & Oral & combate dolores reumáticos. & 9 & 0,09 \\
\hline "culén" & $\begin{array}{l}\text { toda la } \\
\text { planta }\end{array}$ & Infusión & Oral & acción antidiarreica y digestiva & 42 & 0,44 \\
\hline "romero" & $\begin{array}{l}\text { flores y } \\
\text { hojas }\end{array}$ & $\begin{array}{l}\text { Infusión, } \\
\text { Cocimiento }\end{array}$ & Oral & acción anticancerosa y antidiarréica & 4 & 0,04 \\
\hline $\begin{array}{l}\text { "zarzamora } \\
\text { " }\end{array}$ & tallo y hojas & Cocimiento & Oral & $\begin{array}{l}\text { fortalecimiento de las encías, } \\
\text { refrescante. }\end{array}$ & 2 & 0,02 \\
\hline :aúco" & $\begin{array}{l}\text { toda la } \\
\text { planta }\end{array}$ & $\begin{array}{l}\text { Infusión, } \\
\text { Cocimiento }\end{array}$ & Oral & $\begin{array}{l}\text { acción desinflamante, antiséptico y } \\
\text { depurativa. }\end{array}$ & 26 & 0,27 \\
\hline
\end{tabular}




\begin{tabular}{|c|c|c|c|c|c|c|c|c|}
\hline & $\begin{array}{l}\text { Sanguisorba minor Scop. } \\
\text { ROSACEAE }\end{array}$ & "pimpinela" & tallo y hojas & Cocimiento & Oral & $\begin{array}{l}\text { hemostática, contra afecciones del } \\
\text { corazón, antidiarreico, antiséptico, } \\
\text { contra la gastritis. }\end{array}$ & 16 & 0,17 \\
\hline 5 & $\begin{array}{l}\text { Schinus molle L. } \\
\text { ANACARDIACEAE }\end{array}$ & "molle" & $\begin{array}{l}\text { hoja y } \\
\text { corteza }\end{array}$ & $\begin{array}{l}\text { Infusión, } \\
\text { Cocimiento }\end{array}$ & Oral & $\begin{array}{l}\text { acción antiasmática,carminativa y } \\
\text { sedante }\end{array}$ & 43 & 0,45 \\
\hline & $\begin{array}{l}\text { Sicana odorifera (Vell.) } \\
\text { Naudin CUCURBITACEAE }\end{array}$ & "secana" & fruto & Otros & Tópica & acción somnífera y sedante. & 33 & 0,34 \\
\hline & $\begin{array}{l}\text { Solanum tuberosum } \mathrm{L} \text {. } \\
\text { SOLANACEAE }\end{array}$ & "рара" & tubérculo & $\begin{array}{l}\text { Cocimiento, } \\
\text { emplasto, } \\
\text { extracto }\end{array}$ & $\begin{array}{l}\text { Oral y } \\
\text { tópica }\end{array}$ & $\begin{array}{l}\text { acción antidiarreica,colagoga y } \\
\text { digestiva, antigripal y diurético. }\end{array}$ & 57 & 0,59 \\
\hline 5 & $\begin{array}{l}\text { Spartium junceum L. } \\
\text { FABACEAE }\end{array}$ & "retama" & flor & $\begin{array}{l}\text { Infusión, } \\
\text { Cocimiento }\end{array}$ & Baño & acción desinflamante, susto & 8 & 0,08 \\
\hline & $\begin{array}{l}\text { Stevia rebaudiana (Bertoni) } \\
\text { Bertoni } \\
\text { ASTERACEAE }\end{array}$ & "estevia" & hoja & Infusión. & Oral & acción antidiabética. & 20 & 0,21 \\
\hline & $\begin{array}{l}\text { Tagetes filifolia Lag. } \\
\text { ASTERACEAE }\end{array}$ & $\begin{array}{l}\text { "anis } \\
\text { serrano" }\end{array}$ & $\begin{array}{l}\text { toda la } \\
\text { planta }\end{array}$ & Infusión. & Oral & $\begin{array}{l}\text { acción antidiarreica,carminativa y } \\
\text { digestiva }\end{array}$ & 9 & 0,09 \\
\hline & $\begin{array}{l}\text { Tagetes pusilla Kunth } \\
\text { ASTERACEAE }\end{array}$ & $\begin{array}{c}\text { "anis } \\
\text { serrano" }\end{array}$ & $\begin{array}{l}\text { toda la } \\
\text { planta }\end{array}$ & $\begin{array}{l}\text { Infusión, } \\
\text { Cocimiento }\end{array}$ & Oral & $\begin{array}{l}\text { acción antidiarreica,carminativa y } \\
\text { digestiva }\end{array}$ & 10 & 0,10 \\
\hline & $\begin{array}{l}\text { Taraxacum campylodes } \\
\text { G.E.Haglund } \\
\text { ASTERACEAE }\end{array}$ & $\begin{array}{l}\text { "diente de } \\
\text { león", } \\
\text { "amargón" }\end{array}$ & hoja & Cocimiento. & Oral & $\begin{array}{l}\text { contra afecciones biliares, de la } \\
\text { vesícula, antidiarreica, depurativa } \\
\text { de la sangre, colagoga y colerética. }\end{array}$ & 44 & 0,46 \\
\hline & $\begin{array}{l}\text { Uncaria tomentosa (Willd. ex } \\
\text { Schult.) DC. } \\
\text { RUBIACEAE }\end{array}$ & $\begin{array}{l}\text { "uña de } \\
\text { gato" }\end{array}$ & corteza & Cocimiento & Oral & acción carminativa y emenagoga & 63 & 0,66 \\
\hline & $\begin{array}{l}\text { Urtica dioica } \mathrm{L} \text {. } \\
\text { URTICACEAE }\end{array}$ & "ortiga" & hoja & Cocimiento & $\begin{array}{l}\text { Oral, } \\
\text { Tópica }\end{array}$ & $\begin{array}{l}\text { acción antialérgica, antirreunática, } \\
\text { acción purificadora de sangre, } \\
\text { hipertensora. }\end{array}$ & 33 & 0,34 \\
\hline & $\begin{array}{l}\text { Urtica magellanica Juss. ex } \\
\text { Poir. URTICACEAE }\end{array}$ & "ortiga" & hoja & Cocimiento & $\begin{array}{l}\text { Oral, } \\
\text { Tópica }\end{array}$ & $\begin{array}{l}\text { acción antialérgica, antirreunática, } \\
\text { acción purificadora de sangre, } \\
\text { hipertensora. }\end{array}$ & 33 & 0,34 \\
\hline 0 & $\begin{array}{l}\text { Valeriana officinalis L. } \\
\text { CAPRIFOLIACEAE }\end{array}$ & "valeriana" & $\begin{array}{l}\text { raíces } \\
\text { tuberosas }\end{array}$ & $\begin{array}{l}\text { Infusión, } \\
\text { cocimiento }\end{array}$ & Oral & $\begin{array}{l}\text { combate el estrés,contra el dolor de } \\
\text { cabeza, histerias, esquizofrenia e } \\
\text { insomnio. }\end{array}$ & 43 & 0,45 \\
\hline 6 & $\begin{array}{l}\text { Xanthium catharticum Kunth } \\
\text { ASTERACEAE }\end{array}$ & $\begin{array}{l}\text { "juan } \\
\text { alonso" }\end{array}$ & tallo y hojas & Cocimiento & Oral & $\begin{array}{l}\text { acción desinflamante del aparato } \\
\text { reproductor, contra cólicos } \\
\text { mestruales. }\end{array}$ & 24 & 0,25 \\
\hline$\hat{s}$ & $\begin{array}{l}\text { Zingiber officinale Roscoe } \\
\text { ZINGEBERACEAE }\end{array}$ & "kión" & zomas & $\begin{array}{l}\text { extracto, } \\
\text { cocimiento }\end{array}$ & Oral & $\begin{array}{l}\text { acción antiasmática, antidiarreico, } \\
\text { digestiva. }\end{array}$ & 43 & 0,45 \\
\hline
\end{tabular}

Tabla 2

Categorización de las enfermedades y/o dolencias reportadas por el poblador del distrito de Laredo, Trujillo, La Libertad

\begin{tabular}{|c|c|c|c|c|c|c|c|c|c|}
\hline \multirow[t]{2}{*}{$\mathrm{N}$} & \multirow[t]{2}{*}{ Categoría de enfermedad } & \multicolumn{2}{|l|}{$\begin{array}{l}\text { Enfermedad y/o dolencia reportada por los } \\
\text { informantes }\end{array}$} & \multirow[t]{2}{*}{$\begin{array}{l}\mathrm{N}^{\circ} \\
\text { reportes } \\
\text { de uso }\end{array}$} & \multicolumn{3}{|c|}{ Efectividad } & \multirow{2}{*}{$\begin{array}{l}\mathrm{N}^{\circ} \text { de } \\
\text { especies } \\
\text { citadas }\end{array}$} & \multirow[t]{2}{*}{$\mathrm{FCl}$} \\
\hline & & Dolencia y/o enfermedad & $\mathrm{N}$ & & Cur. & Aliv. & Emp. & & \\
\hline 1 & $\begin{array}{l}\text { Enfermedades del sistema } \\
\text { reproductivo }\end{array}$ & $\begin{array}{l}\text { Próstata, cólicos menstruales, dolor de } \\
\text { ovarios, menopausia. }\end{array}$ & 4 & 42 & 7 & 35 & 0 & 12 & 0,73 \\
\hline 2 & $\begin{array}{l}\text { Enfermedades del sistema } \\
\text { respiratorio }\end{array}$ & $\begin{array}{l}\text { Asma, gripe, resfríos, bronquitis, dolor de } \\
\text { garganta, amigdalitis, neumonia. }\end{array}$ & 7 & 54 & 49 & 5 & 0 & 15 & 0,74 \\
\hline 3 & $\begin{array}{l}\text { Enfermedades del sistema } \\
\text { digestivo y gastrointestinal }\end{array}$ & $\begin{array}{l}\text { Dolor de estómago, hígado graso, gastritis, } \\
\text { hemorroides, colitis, úlceras gástricas, gases } \\
\text { intestinales, estreñimiento, colon. }\end{array}$ & 9 & 110 & 87 & 23 & 0 & 24 & 0,79 \\
\hline 4 & $\begin{array}{l}\text { Enfermedades del sistema } \\
\text { cardiovascular }\end{array}$ & $\begin{array}{l}\text { Colesterol, mal de la sangre, mala circulación } \\
\text { sanguínea, obesidad, hipertensión arterial, } \\
\text { corazón. }\end{array}$ & 6 & 54 & 48 & 6 & 0 & 14 & 0,75 \\
\hline 5 & $\begin{array}{l}\text { Enfermedades crónico- } \\
\text { degenerativas }\end{array}$ & Diabetes & 1 & 24 & 0 & 24 & 0 & 8 & 0,70 \\
\hline 6 & Enfermedades auto-inmunes & Alergias & 1 & 2 & 0 & 2 & 0 & 3 & $-1,00$ \\
\hline 7 & $\begin{array}{l}\text { Enfermedades del Sistema } \\
\text { Músculo-esquelético. }\end{array}$ & $\begin{array}{l}\text { Artritis, reumatismo, dolor muscular, dolor de } \\
\text { huesos, osteoporosis. }\end{array}$ & 5 & 35 & 3 & 32 & 0 & 8 & 0,79 \\
\hline 8 & $\begin{array}{l}\text { Síntomas o padecimientos } \\
\text { generales }\end{array}$ & $\begin{array}{l}\text { Dolor de muela, dolor de oído, problemas de } \\
\text { visión. }\end{array}$ & 3 & 13 & 11 & 2 & 0 & 4 & 0,75 \\
\hline 9 & $\begin{array}{l}\text { Enfermedades del sistema } \\
\text { nervioso }\end{array}$ & $\begin{array}{l}\text { Ansiedad, insomnio, dolor de cabeza, } \\
\text { Parkinson, Nerviosismo. }\end{array}$ & 5 & 47 & 39 & 8 & 0 & 11 & 0,78 \\
\hline 10 & $\begin{array}{l}\text { Enfermedades del sistema } \\
\text { urinario }\end{array}$ & $\begin{array}{l}\text { Problemas de las vías urinarias, problemas del } \\
\text { riñón y vejiga, cálculos renales. }\end{array}$ & 4 & 56 & 56 & 0 & 0 & 15 & 0,75 \\
\hline 11 & Enfermedades de la piel & $\begin{array}{l}\text { Acné, problemas de la piel, heridas en la piel, } \\
\text { micosis. }\end{array}$ & 4 & 8 & 2 & 6 & 0 & 12 & $-0,57$ \\
\hline & & TOTAL & 49 & 444 & 302 & 143 & & & \\
\hline & & CENTAJE (\%) & & 100 & 68,02 & 32,08 & & & \\
\hline
\end{tabular}




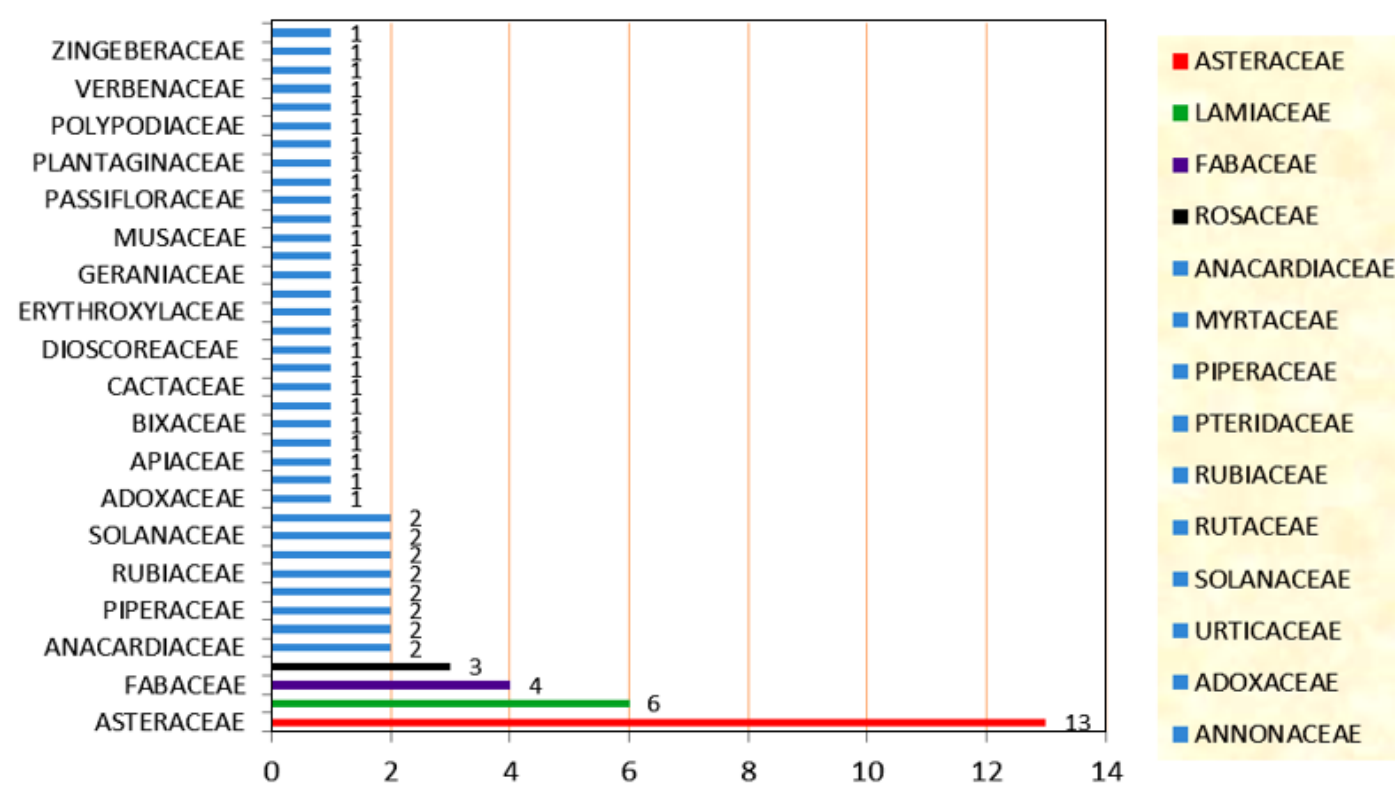

Figura 2. Familias más representativas de flora utilizada por el poblador del distrito de Laredo, Trujillo, La Libertad.

Tabla 5

Estado de salud desde la percepción del poblador del distrito de Laredo, según las dimensiones Rol Físico, Rol Emocional, Salud Mental, Vitalidad y Función Social

\begin{tabular}{|c|c|c|c|c|c|c|c|c|c|c|c|}
\hline \multirow{2}{*}{ DIMENSIÓN } & \multirow{2}{*}{ Efectividad } & \multicolumn{2}{|c|}{ Siempre } & \multicolumn{2}{|c|}{ Casi } & \multicolumn{2}{|c|}{ Algunas } & \multicolumn{2}{|c|}{ Sólo } & \multicolumn{2}{|c|}{ Nunca } \\
\hline & & $\mathrm{N}$ & $\%$ & $\mathrm{~N}$ & $\%$ & $\mathrm{~N}$ & $\%$ & $\mathrm{~N}$ & $\%$ & $\mathrm{~N}$ & $\%$ \\
\hline $\begin{array}{l}\text { ROL FISICO } \\
\text { Hizo menos de lo deseado a causa de }\end{array}$ & 96 & 0 & 0 & 0 & 0,00 & 28 & 29,17 & 17 & 17,71 & 51 & 53,13 \\
\hline $\begin{array}{l}\text { Dejó de hacer tareas a causa de su } \\
\text { salud física }\end{array}$ & 96 & 0 & 0 & 0 & 0,00 & 28 & 29,17 & 26 & 27,08 & 42 & 43,75 \\
\hline MEDIA & & & & & 0,00 & & 29,17 & & 22,40 & & 48,44 \\
\hline TOTAL PORCENTAJE POSITIVO & & 22 & $\%+48$ & $4 \%=$ & $70,83 \%$ & & & & & & \\
\hline $\begin{array}{l}\text { ROL EMOCIONAL } \\
\text { Hizo menos de lo deseado por alqún }\end{array}$ & 96 & 0 & 0 & 11 & 11,46 & 19 & 19,79 & 32 & 33,33 & 34 & 35,42 \\
\hline $\begin{array}{l}\text { Hizo sus tareas menos cuidadosamente } \\
\text { por alqún problema emocional }\end{array}$ & 96 & 0 & 0 & 7 & 7,29 & 28 & 29,17 & 28 & 29,17 & 32 & 33,33 \\
\hline MEDIA & & & & & 9,38 & & 24,48 & & 31,25 & & 34,38 \\
\hline TOTAL PORCENTAJE POSITIVO & & & $5 \%+34$ & $88 \%=$ & $65,63 \%$ & & & & & & \\
\hline $\begin{array}{l}\text { SALUD MENTAL } \\
\text { Se sintió calmado y tranquilo }\end{array}$ & 96 & 40 & 41,67 & 32 & 33,33 & 24 & 25,00 & 0 & 0,00 & 0 & 0,00 \\
\hline Se sintió animado y positivo & 96 & 32 & 33,33 & 42 & 43,75 & 22 & 22,92 & 0 & 0,00 & 0 & 0,00 \\
\hline $\begin{array}{l}\text { MEDIA } \\
\text { TOTAL PORCENTAJE POSITIVO }\end{array}$ & & & $\frac{37,5}{0+38,5}$ & - & 38,54 & & 23,96 & & & & \\
\hline $\begin{array}{l}\text { VITALIDAD } \\
\text { Tuvo mucha eneraía }\end{array}$ & 96 & 32 & 33,33 & 39 & 40,63 & 25 & 26,04 & 0 & 0,00 & 0 & 0,00 \\
\hline MEDIA & & & 33,33 & & 40,63 & & 26,04 & & & & \\
\hline TOTAL PORCENTAJE POSITIVO & & & $3 \%+40$ & $3 \%=$ & 73,96 & & & & & & \\
\hline $\begin{array}{l}\text { FUNCIÓN SOCIAL } \\
\text { Tuvo dificultades para cumplir con sus }\end{array}$ & 96 & 0 & 0 & 4 & 4,17 & 19 & 19,79 & 27 & 28,13 & 46 & 47,92 \\
\hline $\begin{array}{l}\text { MEDIA } \\
\text { TOTAL PORCENTAJE POSITIVO }\end{array}$ & & 47 & $2 \%+28$ & $3 \%=$ & $\begin{array}{l}4,17 \\
6,05\end{array}$ & & 19,79 & & 28,13 & & 47,92 \\
\hline
\end{tabular}

Se concluye que el $68,02 \%$ de la población se "curó"; mientras que un $32,08 \%$ se "alivió"; infiriéndose de estos resultados que las plantas medicinales tienen una efectividad del $100 \%$ ya que ningún poblador manifestó haber mantenido igual o empeorado su salud por el uso de las mismas; pues sólo se curaron y 10 aliviaron; hallazgos que si concuerdan con Bocanegra et al. (2011), quienes manifiestan que la efectividad de las plantas medicinales en Curgos, Sánchez Carrión, Perú; es de un $96,81 \%$; reflejadas en un $37,31 \%$ de personas curadas y un $59,50 \%$ de personas aliviadas y sumado a ello, aunque no con detalles estadísticos autores como Bussmann y Sharon (2006, 2015) Vera y Sánchez (2016), Castañeda y Albán (2016), Mostacero et al. (2019) resaltan la efectividad de las plantas medicinales en la cura de diversas enfermedades en el Perú. 
Tabla 6

Estado de salud del poblador del distrito de Laredo, según las dimensiones Dolor Corporal

\begin{tabular}{|c|c|c|c|c|c|c|c|c|c|c|c|}
\hline \multirow{2}{*}{ Dimensión } & \multirow{2}{*}{ Efectividad } & \multicolumn{2}{|c|}{ Nada } & \multicolumn{2}{|c|}{ Un poco } & \multicolumn{2}{|c|}{ Regular } & \multicolumn{2}{|c|}{ Bastante } & \multicolumn{2}{|c|}{ Mucho } \\
\hline & & $\mathrm{N}$ & $\%$ & $\mathrm{~N}$ & $\%$ & $\mathrm{~N}$ & $\%$ & $\mathrm{~N}$ & $\%$ & $\mathrm{~N}$ & $\%$ \\
\hline DOLOR CORPORAL & & & & & & & & & & & \\
\hline $\begin{array}{l}\text { Hasta que punto el dolor le } \\
\text { dificulto hacer sus } \\
\text { actividades }\end{array}$ & 96 & 41 & 42,71 & 35 & 36,46 & 20 & 20,83 & 0 & 0,00 & 0 & 0,00 \\
\hline MEDIA & & & 42,71 & & 36,46 & & 20,83 & & & & \\
\hline TOTAL porcentaje positivo & & 42,7 & $+36,46$ & 79,1 & & & & & & & \\
\hline
\end{tabular}

Referente a la calidad de vida, que obtuvo el poblador de Laredo, después del uso de las plantas medicinales, y tal como se analiza en la Tabla 7, ésta se vio altamente beneficiada; pues luego del tratamiento, el $77,41 \%$ de la población refiere haber mejorado su calidad de vida; en cada una de las dimensiones o escalas establecidas por Ware et al. (1993) y Ware et al. (1996). De allí que, desde la perspectiva del poblador del distrito de Laredo, en cuanto a las dimensiones referidas en la Tabla 3, la Salud General, se benefició en un $80,2 \%$; la Función Física, presentada en la Tabla 4, en un $97,39 \%$. El Rol Físico, Rol Emocional, Salud Mental, Vitalidad y Función Social, referida en la Tabla 5 , el poblador se benefició en un $70,83 \%, 65,63 \%, 76,04 \%, 73,96 \%$ y $76,05 \%$ respectivamente y el Dolor Corporal, referido en la Tabla 6, se benefició en un $79,17 \%$.

Tabla 7

Calidad de vida según la percepción del poblador del distrito de Laredo, Trujillo

\begin{tabular}{clc}
\hline \multicolumn{2}{c}{ Calidad De Vida } \\
\hline $\mathrm{N}^{\circ}$ & \multicolumn{1}{c}{ Dimensión } & Porcentaje Positivo \\
\hline 1 & Salud general & 80,2 \\
2 & Función física & 97,39 \\
3 & Rol físico & 70,83 \\
4 & Rol emocional & 65,63 \\
5 & Salud mental & 76,04 \\
6 & Vitalidad & 73,96 \\
7 & Función social & 76,05 \\
8 & Dolor corporal & 79,17 \\
\hline \multicolumn{2}{c}{ Media } \\
\hline
\end{tabular}

Estos resultados sobre la calidad de vida se asemejan a los obtenidos por Bocanegra et al. (2011); quienes demostraron la mejora de la calidad de vida por el uso de las plantas medicinales en Curgos; y de manera general, con los trabajos de Bussmann y Sharon (2015), Castañeda y Albán (2016), Lara et al. (2019), Mostacero et al. (2019), que también dejan establecido que el uso de las plantas medicinales mejoran la calidad de vida del poblador, debido a la efectividad que poseen.

\section{Conclusiones}

En el distrito de Laredo se utilizan 68 especies etnomedicinales; las que están distribuidas en 67 géneros y 38 familias. Donde las Asteraceae (13 especies), Lamiaceae (6 especies), Fabaceae (4 especies) y Rosaceae (3 especies) son las más representativas por el número de especies.

La efectividad de la medicina herbolaria en Laredo alcanzó el 100\%; dado a que un $68,02 \%$ se "curan" y el 32,08 \% se "alivian".

La mejora de la Calidad de vida, por el uso de la medicina herbolaria, desde la perspectiva del poblador de Laredo, alcanzó al 77,41 \% de la población.

Las especies medicinales mayormente utilizadas son: Gentianella bicolor "corpus huay"; Matricaria chamomilla "manzanilla"; Uncaria tomentosa "uña de gato"; Linum usitatissimum "linaza"; Solanum tuberosum "papa"; Equisetum bogotense "cola de caballo"; Opuntia ficus-indica "tuna" y Aloe vera "sábila".

Los pobladores del distrito de Laredo sufren de 49 enfermedades $\mathrm{y} / \mathrm{o}$ dolencias diferentes; los mismos que han sido agrupadas en 11 categorías y donde prevalecen, las enfermedades de los Sistemas Músculo-esquelético, digestivo y gastrointestinal, nervioso, cardiovascular, urinario, Padecimientos Generales, respiratorio, reproductivo y del grupo de las crónico-degenerativas.

\section{ORCID \\ J. Mostacero-León (D) https://orcid.org/0000-0003-2556-3013 \\ A. De La Cruz-Castillo (D) https://orcid.org/0000-0002-5409-6146 \\ E. Lopéz-Medina (DD https://orcid.org/0000-0001-7719-8607 \\ A. Gil-Riveroa (D) https://orcid.org/0000-0002-4521-5588}

\section{Referencias bibliográficas}

Annan, K.; Houghton, P. 2007. Antibacterial, Antioxidant and Fibroblast Growth Stimulation of Aqueous Extracts of Ficus asperifolia Miq. and Gossypium arboreum L., Wound-healing Plants of Ghana. Journal of Ethnopharmacology, 119: 141-144.

Bocanegra, L.; Espinola, G.; Mostacero, J. 2009. Viabilidad y fiabilidad del cuestionario SF-12 V2 modificado y aplicado en pobladores de la sierra de La Libertad. Congreso Internacional de Ecología y Medio Ambiente; Trujillo, Perú. 
Bocanegra, L.; Bocanegra, F.; Mostacero, J. 2011. Efectividad de la medicina herbolaria y su impacto en la calidad de vida de los pobladores de Curgos, Perú. UCV - Scientia 3(1): 23-34.

Bradburn, N. 1969. The Structure of psychological Well-Being Chicago: Aldine

Brako, L.; Zarucchi, J. 1993. Catálogo de las Angiospermas y Gimnospermas del Perú. Monogr. Syst. Bot. Missouri Bot. Garden. Vol 45

Bussmann, R.; Sharon, D. 2006. Traditional medicinal plant use in Northern Peru: tracking two thousand years of healing culture. Journal of Ethnobiology and Ethnomedicine 2(1): $47: 74$

Bussmann, R.; Glenn, A. 2010. Medicinal plants used in Peru for the treatment of respiratory disorders. Rev. peru. biol. 17(2): 331 346.

Bussmann, R.; Sharon, D. 2015. Plantas medicinales de Los Andes y la Amazonia - La Flora mágica y medicinal del Norte del Perú. Graficart SRL, Trujillo, Perú.

Castañeda, R.; Albán, J. 2016. Importancia cultural de la flora silvestre del distrito de Pamparomás, Ancash, Perú. Ecol. apl. 15(2): 151-169.

Castillo, H.; Cochachin, E.; Albán, J. 2017. Plantas comercializadas por herbolarios en el mercado del distrito de Cajabamba (Cajamarca, Perú). Bol Latinoam Caribe Plant Med Aromat 16(3): 303-318.

Centro de investigación, promoción y asesoramiento para el desarrollo de Laredo. (CIPADEL). 1992. Historia, Geografía y Economía de Laredo. Trujillo-Perú

Consiglio, E.; Belloso, W. 2003. Nuevos indicadores clínicos: La calidad de vida relacionada con la salud. Medicina (Buenos Aires) 63(2):172-178

De la Cruz, A.; Mostacero, J. 2019. Uso de plantas medicinales para la cura de enfermedades y/o dolencias: El caso del poblador de la provincia de Trujillo, Perú. Revista de Investigación Científica Universidad Nacional de Tumbes, Perú. Manglar 16(2): 119124

Fernández, J.; Fernández, M.; Cieza, A. 2010. Los conceptos de calidad de vida, salud y bienestar analizados desde la perspectiva de la Clasificación Internacional del Funcionamiento (CIF). Rev. Española de Salud Pública 84(2): 169-184.

Garzón, P. 2016. Conocimiento Tradicional sobre las plantas medicinales de yarumo (Cecropia sciadophylla), carambolo (Averrhoa carambola) y uña de gato (Uncaria tomentosa) en el resguardo indígena de Macedonia, Amazonas. revista.luna.azúl; 43: 386-414

Glatzer, E.; Zapf, W. 1984. Lebesqualität in der Bundesrepublik. Darmstadt: Wissenchaftliche Buchgesellschaft.

Heinrich, M.; Edwards, S: Moerman, E.; Leonti, M. 2009 Ethnopharmacological field studies: a critical assessment of their conceptual basis and methods. Journal of Ethnopharmacol 124: $1-17$

Hurtado, J.; Albán, J. 2018. Conocimiento tradicional de la flora silvestre en las comunidades campesinas del Santuario Histórico de la Pampa de Ayacucho (Quinua, Ayacucho, Perú). Bol Latinoam Caribe Plant Med Aromat 17(3): 286-301.

Huamantupa, I.; Cuba, M.; Urrunaga, R.; Paz, E.; Ananya, N.; Callalli, M.; Pallqui, N.; Coasaca, H. 2011. Riqueza, uso y origen de plantas medicinales expendidas en los mercados de la ciudad del Cusco. Rev Peru Biol 18(3): 283-291.

ICD-(International Statistical Classification of Diseases and Related Health Problems (World Health Organization) (ICD). Disponible en: http://www.who.int/classifications/apps/icd/icd10online

Lara, E.; Fernández, E.; Zepeda, J.; Lara, D.; Aguilar, A.; VanDamme, P. 2019. Etnomedicina en Los Altos de Chiapas México. Bol Latinoam Caribe Plant Med Aromat 18(1): 42-57.
Mostacero, J.; Mejía, F.; Zelada, W.; Medina, C. 2007. Biogeografía del Perú. Asamblea Nacional de Rectores. Trujillo - Perú.

Mostacero, J.; Mejía, F.; Gamarra, O. 2009. Fanerógamas del Perú: Taxonomía, utilidad y ecogeografía. CONCYTEC. Trujillo - Perú.

Mostacero, J.; Castillo, F.; Mejía, F.; Gamarra, O.; Charcape, J.; Ramírez, R. 2011. Plantas Medicinales del Perú: Taxonomía, Ecogeografía, Fenología y Etnobotánica. Trujillo - Perú: Asamblea Nacional de Rectores Fondo Editorial.

Mostacero, J.; Peláez, F.; Alarcón, N.; De La Cruz, A.; Alva, R.; Charcape, M. 2019. Plantas utilizadas para el tratamiento del cáncer expendidas en los principales mercados de la provincia de Trujillo, Perú, 2016 - 2017. Bol Latinoam Caribe Plant Med Aromat 18(1): 81-94.

Ortega-Cala, L.; Monroy-Ortiz, C.; Monroy-Martínez, R.; ColínBahena, O.; Flores-Franco, G.; Luna-Cavazos, M.; MonroyOrtiz, R. 2019. Plantas medicinales utilizadas para enfermedades del sistema digestivo en Tetela del Volcán, Estado de Morelos, México. Boletín Latinoamericano y del Caribe de plantas medicinales y aromáticas 18(2):106-129.

Pascual, D.; Pérez, Y.; Morales, I.; Castellanos, I.; González, H. 2014. Algunas consideraciones sobre el surgimiento y la evolución de la medicina natural y tradicional. MEDISAN 18(10): 1467-74.

Peláez, E.; Yuto, J. 2001. La Juventud y su participación ciudadana en la Ciudad de Laredo. Informe final de Prácticas Preprofesionales de Antropología Social. Tesis de pregrado, Universidad Nacional de Trujillo. Perú. 143 pp.

Picard, L.; Villard, M. 1982. Metodología Utilizada Para la Introducción de Especies Forestales en Cajamarca - Perú. N3 3 . Edit. Centro de Investigación y Capacitación Forestal. CICAFOR. Cajamarca, Perú.

Pretell, J.; Ocaña, R.; Barahona, E. 1985. Apuntes Sobre Algunas Especies Forestales Nativas de la Sierra Peruana. Proyecto FAO/Holanda/INFOR. Edit. Centauro S.A. Lima, Perú.

Rengifo, E.; Vargas, G. 2013. Physalis angulata L. (Bolsa Mullaca): A Review of its Traditional Uses, Chemistry and Pharmacology. Bol Latinoam Caribe Plant Med Aromat 12(5): 431-445.

Sagástegui, A. 1995. Diversidad Florística de Contumazá. Trujillo Perú: Fondo Editorial Univ. Antenor Orrego. Edit. Libertad.

Salaverry, O.; Cabrera, J. 2014. Florística de algunas plantas medicinales [galería]. Rev. Peru. Med. Exp. Salud Publica 31: 165-168.

Schwartzmann, L. 2003. Calidad de vida relacionada con la salud: aspectos conceptuales. Cienc. Enferm. 9(2):9-21.

Vera, B.; Sánchez, M. 2016. Plantas medicinales y predictibilidad de uso en algunas veredas del corregimiento de San Cristóbal (Antioquia), Colombia. Actu. Biol 38(105): 167-180.

Vilagut, G.; Ferrer, M.; Rajmil, L.; Rebollo, P.; Permanyer-Miralda, G.; Quintana, J.; Santed, R.; Valderas, J.; Ribera, A.; DomingoSalvany, A.; Alonsoa, J. 2005. El Cuestionario de Salud SF-36 español: una década de experiencia y nuevos desarrollos. Gac Sanit 19(2):135-50.

Ware, J.; Sherbourne, C. 1992. The MOS 36-item short-form health survey (SF-36) I. Conceptual framework and item selection. Med Care. 30: 473-83

Ware, J.; Snow, K.; Kosinski, M.; Gandek, B. 1993. SF-36 Health Survey: manual and interpretation guide. Boston: New England Medical Center.

Ware, J.; Kosinski, M.; Keller, S. 1996. A 12-Item Short-Form Health Survey: construction of scales and preliminary tests of reliability and validity. Med Care 34: 220-33.

Zambrano, L.; Buenaño, M.; Mancera, N.; Jiménez, E. 2015. Estudio etnobotánico de plantas medicinales utilizadas por los habitantes del área rural de la Parroquia San Carlos, Quevedo, Ecuador. Rev. Univ. Salud 17: 97-111. 